\title{
Risk factors for surgical site infection and association of surgical site infection with survival of lower rectal cancer patients without clinical lateral pelvic lymph node metastasis (clinical Stage II/III): Analysis of data from JCOG0212
}

\author{
Kenji Katsumata ${ }^{1}$ (1) Masanobu Enomoto ${ }^{1} \cdot$ Tetsuo Ishizaki $^{1} \cdot$ Shin Fujita ${ }^{2} \cdot$ Yukihide Kanemitsu $^{3} \cdot$ Masaaki Ito $^{4}$. \\ Akio Shiomi ${ }^{5} \cdot$ Koji Komori $^{6} \cdot$ Masayuki Ohue $^{7} \cdot$ Mitsuyoshi Ota $^{8} \cdot$ Yoshihiro Akazai $^{9} \cdot$ Manabu Shiozawa $^{10}$. \\ Takashi Yamaguchi $^{11}$ • Hiroyuki Bando ${ }^{12}$. Mitugu Sekimoto ${ }^{13} \cdot$ Takaya Kobatake $^{14} \cdot$ Ryunosuke Machida $^{15}$. \\ Takayuki Akasu $^{16}$. Yoshihiro Moriya ${ }^{17}$. the Colorectal Cancer Study Group of Japan Clinical Oncology Group
}

Received: 30 March 2021 / Accepted: 24 June 2021 / Published online: 18 August 2021

(c) The Author(s) 2021

\begin{abstract}
This study aimed to examine the risk factors for surgical site infection (SSI) and the association of that with recurrence in JCOG0212. The results for secondary endpoints showed that compared with the mesorectal excision (ME) alone group, ME with lateral lymph node dissection (LLND) group showed significantly longer operative time and significantly higher blood loss. These results suggested that LLND was a risk factor for SSI. All 701 patients registered in JCOG0212 were analyzed in this study. Wound infection was defined as incisional/deep SSI, and pelvic abscess and anastomotic leakage were defined as organ/space SSI. The risk factors for the incidence of SSI and the effect of SSI on relapse-free survival (RFS) were investigated. Multivariable odds ratio of Grade 2 or higher all SSI was 0.58 [95\% Confidence interval: 0.36-0.93] for female (vs. male) and that of Grade 2 or higher incisional/deep SSI was 2.24 [1.03-4.86] for blood infusion. For RFS, patients with Grade 3 or higher all SSI showed poor prognosis (multivariable hazard ratio: 1.66 [1.03-2.68]). LLND is not significant factor for the incidence of all SSI. Male sex might be a risk factor of Grade 2 or higher SSI, and blood transfusion is a possible risk factor of Grade 2 or higher incisional/deep SSI. Grade 3 or higher all SSI might be a significant worse prognostic factor for lower rectal cancer.
\end{abstract}

Keywords Rectal cancer · Latera lymph node dissection · Surgical site infection · Recurrence

$\begin{array}{ll}\text { Abbreviations } \\ \text { JCOG 0212 } & \begin{array}{l}\text { Mesorectal excision with or without lateral } \\ \text { lymph node dissection for Clinical stage II/ }\end{array} \\ & \begin{array}{l}\text { III lower rectal cancer, a multicenter, rand- } \\ \text { omized controlled, noninferiority trial }\end{array} \\ \text { CI } & \text { Confidence intervals } \\ \text { LLND } & \text { Lateral lymph node dissection } \\ \text { ME } & \text { Mesorectal excision } \\ \text { OR } & \text { Odds ratio } \\ \text { RFS } & \text { Recurrence-free survival } \\ \text { SSI } & \text { Surgical site infection } \\ \text { WHO } & \text { World Health Organization }\end{array}$

Kenji Katsumata

k-katsu@tokyo-med.ac.jp

Extended author information available on the last page of the article

\section{Introduction}

Surgical site infection (SSI), an infection associated with surgery, is a major complication. Generally, SSI is the second-most frequent occurring medical infection in developed countries [1]. One of the issues with the incidence of SSI with cancer surgery is that SSI leads to substantial health economic losses due to prolonged hospitalization [2]. Another issue is that the development of SSI is a poor prognostic factor of cancer [3]. Thus, countermeasures for SSI are important in medical economics and the prognosis of cancer. A wide range of evidence-based guidelines has been developed by the World Health Organization (WHO) [4]. and the Centers for Disease Control and Prevention [5]. In particular, the incidence of wound infection and anastomotic leakage is more frequent in rectal cancer than in other organ cancers [6]. Moreover, as mentioned 
above, many reports indicated that the development of SSI is a risk factor for cancer recurrence; this has also been suggested in colorectal cancer, indicating that this is an important issue [7].

In surgery for the treatment of lower rectal cancer, mesorectal excision (ME) with lateral pelvic lymph node dissection (LLND) is the standard surgical procedure for patients with advanced lower rectal cancer in Japan [8]. Autonomic nerve-sparing surgery with LLND was reportedly useful in low rectal cancer in many retrospective studies [9]. However, a randomized, controlled trial was essential to evaluate the benefits of extended lymphadenectomy for patients without extra-mesenteric metastasis. In order to acquire high-level evidence, the JCOG0212 randomized controlled trial was conducted in Japan.

On the other hand, ME with chemo-radiotherapy is the global standard treatment for rectal cancer. Therefore, we conducted a randomized controlled trial, JCOG0212, and concluded that the non-inferiority of ME alone could not be confirmed compared with ME with LLND [10].

To the best of our knowledge, JCOG0212 may be the only clinical trial worldwide that has been performed on LLND. By performing LLND, recurrences in the pelvis were reduced from 12.6 to $7.4 \%$; however, the duration of surgery increased by $106 \mathrm{~min}$, the volume of blood loss increased by $239 \mathrm{~mL}$, and Grade 3 or higher complications were reported in $22 \%$ versus $16 \%$ of patients [11]. Generally, prolongation of the operative time and increase of the bleeding are major factors of the incidence of SSI. Even when these negative factors were considered, the results showed that LLND was the standard procedure performed. However, the issue of whether these negative aspects of LLND have any effect on SSI or on recurrence at the very least remained unresolved. Thus, in the present study, whether risk factors for the incidence of SSI and LLND were associated with SSI and whether SSI was a prognostic factor for relapse-free survival (RFS) were analyzed using data from the JCOG0212.

\section{Materials and methods}

\section{Population}

Inclusion criteria of JCOG0212 have been reported previously. Eligibility criteria included histologically proven rectal cancer at clinical Stage II or III; (T-factor T2-T3 and N factor regional lymph node except of pelvic lateral and root of inferior mesenteric arterial lymph node), with the lower margin being below the peritoneal reflection; performance status of 0 or 1; and age 20-75 years. A total of 701 patients were randomized to the ME with LLND group $(n=351)$ or the ME alone group $(\mathrm{n}=350)$.

\section{Definitions of endpoints}

Wound infection was defined as incisional/deep SSI, and pelvic abscess and anastomotic leakage were defined as organ/ space SSI according to NCI-CTC ver.2.0 in the present study [12]. The definition of Grade is that Grade 0 is absent, Grade 1 is mild, Grade 2 is moderate, Grade 3 is severe, Grade 4 is life-threatening SSI. Grade 1 is local infection and does not affect a general condition. All SSI included incisional/deep SSI and organ/space SSI. The RFS and local RFS were same definitions in JCOG0212 [13].

\section{Statistical analysis}

Each event numbers were small. Therefore risk factors for the incidence of Grade 2 or higher SSI were analyzed according to definitions of SSI (all SSI, incisional/deep SSI, and organ/space SSI). Odds ratio (OR) and its 95\% confidence intervals (CI) for each factor were calculated by the multivariable logistic regression model in 691 patients without missing variables. The RFS and local RFS by definitions of SSI Grade were estimated according to the Kaplan-Meier method in all 701 patients. The effects of SSI on RFS and local RFS were evaluated by the multivariable Cox regression analysis in 693 patients without missing variables. The covariates were selected using a backward method with exclusion criteria of significance level of 0.20 under forcibly including the presence or absence of Grade 2 or higher and Grade 3 or higher SSI. Hazard ratio (HR) and its 95\% CIs for each factor in the final model were described. A p-value of $\leq 0.05$ was considered statistically significant. All statistical analyses were performed by SAS ver. 9.4.

\section{Results}

\section{Risk factors for incidence of SSI}

The grade 2 or higher all SSI was observed in 120 (17.1\%) patients and all Grade 3 or higher in $48(6.8 \%)$ patients (Table 1). The Grade 2 or higher incisional/deep SSI was observed in 55 (7.8\%) patients and incisional/deep Grade 3 or higher SSI in 18 (2.6\%) patients. Grade 2 or higher organ/ space SSI was observed in $81(11.6 \%)$ patients and Grade 3 or higher organ/space SSI in 32 (4.6\%) patients.

In the multivariable logistic regression, LLND was not associated with any SSI. In other factors, male sex was a significant risk factor for the incidence of all Grade 2 or higher SSI (OR: 0.58, 95\% CI 0.36-0.93, $\mathrm{p}=0.023$ ). Presence of blood transfusions was a significant risk factor for the incidence of Grade 2 or higher incisional/deep SSI (OR: 
Table 1 Incidence of Surgical site infection (SSI)

\begin{tabular}{|c|c|c|c|c|c|c|c|c|c|c|}
\hline SSI & Grade 0 & Grade 1 & Grade 2 & Grade 3 & Grade 4 & $\geq$ Grade 2 & $\geq$ Grade 3 & $\% \geq$ Grade 2 & $\% \geq$ Grade 3 & Total \\
\hline Incisional/Deep & 634 & 12 & 37 & 17 & 1 & 55 & 18 & 7.8 & 2.6 & 701 \\
\hline Organ/space & 604 & 16 & 49 & 30 & 2 & 81 & 32 & 11.6 & 4.6 & 701 \\
\hline $\begin{array}{l}\text { All (Incisional/ } \\
\text { Deep/Organ/ } \\
\text { space) }\end{array}$ & 556 & 25 & 72 & 46 & 2 & 120 & 48 & 17.1 & 6.8 & 701 \\
\hline
\end{tabular}

$2.24,95 \%$ CI $1.03-4.86, p=0.043)$. No significant factors for the incidence of Grade 2 or higher organ/space SSI were noted. (Table 2).

\section{Associations of SSI with RFS and local RFS}

The 5-year RFS for patients with Grade 1 or lower and Grade 2 or higher all SSI were $73.6 \%$ (95\% CI 769.8-77.0\%) and $72.5 \%$ (95\% CI 63.5-79.6\%), respectively (univariable HR: $0.942,95 \%$ CI 0.65-1.33). For incisional/deep SSI, the 5-year RFS for patients with Grade 1 or lower and Grade 2 or higher SSI were $73.4 \%$ (95\% CI 69.8-76.7\%) and 72.6\% (95\% CI 58.8-82.5\%), respectively (univariable HR: 0.906, 95\% CI 0.55-1.51). For organ/space SSI, the 5-year RFS in patients with Grade 1 or lower and Grade 2 or higher SSI were $73.6 \%$ (95\% CI 69.9-76.9\%) and 71.6\% (95\% CI 60.4-80.1\%), respectively (univariate HR: $0.995,95 \%$ CI $0.65-1.52)$.

The 5-year RFS for patients with Grade 2 or lower and Grade 3 or higher all SSI were $74.3 \%$ (95\% CI 70.8-77.5\%) and $60.4 \%$ (95\% CI 45.2-72.6\%), respectively (univariable HR: 1.49 , 95\% CI 0.93-2.39). For incisional/deep SSI, the 5-year RFS for patients with Grade 2 or lower and Grade 3 or higher SSI were 73.7\% (95\% CI 70.2-76.8\%) and 61.1\% (95\% CI 35.3-79.2\%), respectively (univariable HR: 1.45 , 95\% CI 0.68-3.08). For organ/space SSI, the 5-year RFS in patients with Grade 2 or lower and Grade 3 or higher SSI were $74.2 \%$ (95\% CI 70.7-77.3\%) and 56.3\% (95\% CI $37.6-71.3 \%$ ), respectively (univariate HR: $1.71,95 \%$ CI 0.99-2.93) (Fig. 1).

In the multivariable analysis for Grade 3 or higher all SSI, lymph node metastases, blood transfusions, pathological curative degree, pathological T stage, pathological $\mathrm{N}$ stage, and postoperative adjuvant chemotherapy were selected as the covariates in the final model RFS based on stepwise method. Regarding multivariable analyses for incisional/deep SSI and organ/space SSI, histological type for primary lesion was also selected as covariate. Patients with Grade 3 or higher all SSI showed poor RFS (HR: 1.66, 95\% CI 1.03-2.68, $\mathrm{p}=0.038$ ). Incisional/deep Grade 3 or higher SSI was not a significant risk factor (HR: 1.43, 95\% CI 0.66-3.13, $\mathrm{p}=0.367$ ), whereas organ/space SSI was significantly associated with RFS (HR: 2.01, 95\% CI 1.16-3.49, $p=0.013$ ). (Table 3 ). No significant differences were noted in the local RFS for any definitions of SSI. In addition, no large differences in presence or absence of LLND was observed.

\section{Discussion}

This study showed that male sex might be a risk factor of Grade 2 or higher SSI, and blood transfusion is a possible risk factor of Grade 2 or higher incisional/deep SSI. Moreover, Grade 3 or higher SSI might be a significant prognostic factor for RFS in patients with lower rectal cancer. On the contrary, LLND was not associated with the incidence of any SSI, including incisional/deep and organ/space SSI.

The existing recommendation in guidelines such as those established by the WHO for preparation prior to colorectal cancer surgery is to use oral antibiotics and mechanical bowel preparation in conjunction with oral antibiotics the day prior to surgery [4]. At the time of JCOG0212, which was conducted from 2003 to 2010 , it was presumed that many institutions used mechanical bowel preparation alone because intestinal pretreatment with a combination of oral antibiotics given daily for 3 days induced a high proportion of drug resistance. Kobayashi et al. reported that approximately $10 \%$ of patients received antibiotics the day prior to surgery in 2007 [14]. It is necessary to think about incidence of SSI of JCOG0212 in consideration of condition which was not good rather than present status.

Many factors related to SSI have been identified, such as smoking, hyperglycemia, anemia, and ASA score; in addition, male sex and colostomy have been suggested as factors related to SSI in rectal cancer [15]. In the present analyses, blood transfusions and male sex were found to be factors related to SSI. In contrast, for the involvement of LLND, blood transfusions tended to be significantly more prevalent in the TME and LLND groups. The incidence of Grade 3-4 postoperative complications was also higher [4]; however, to the best of our knowledge, in this study, it was demonstrated for the first time that LLND was not a risk factor for incidence of SSI in lower rectal surgery. The frequency of incidence of SSI in colorectal cancer surgery is higher than that in other gastrointestinal cancers, and 


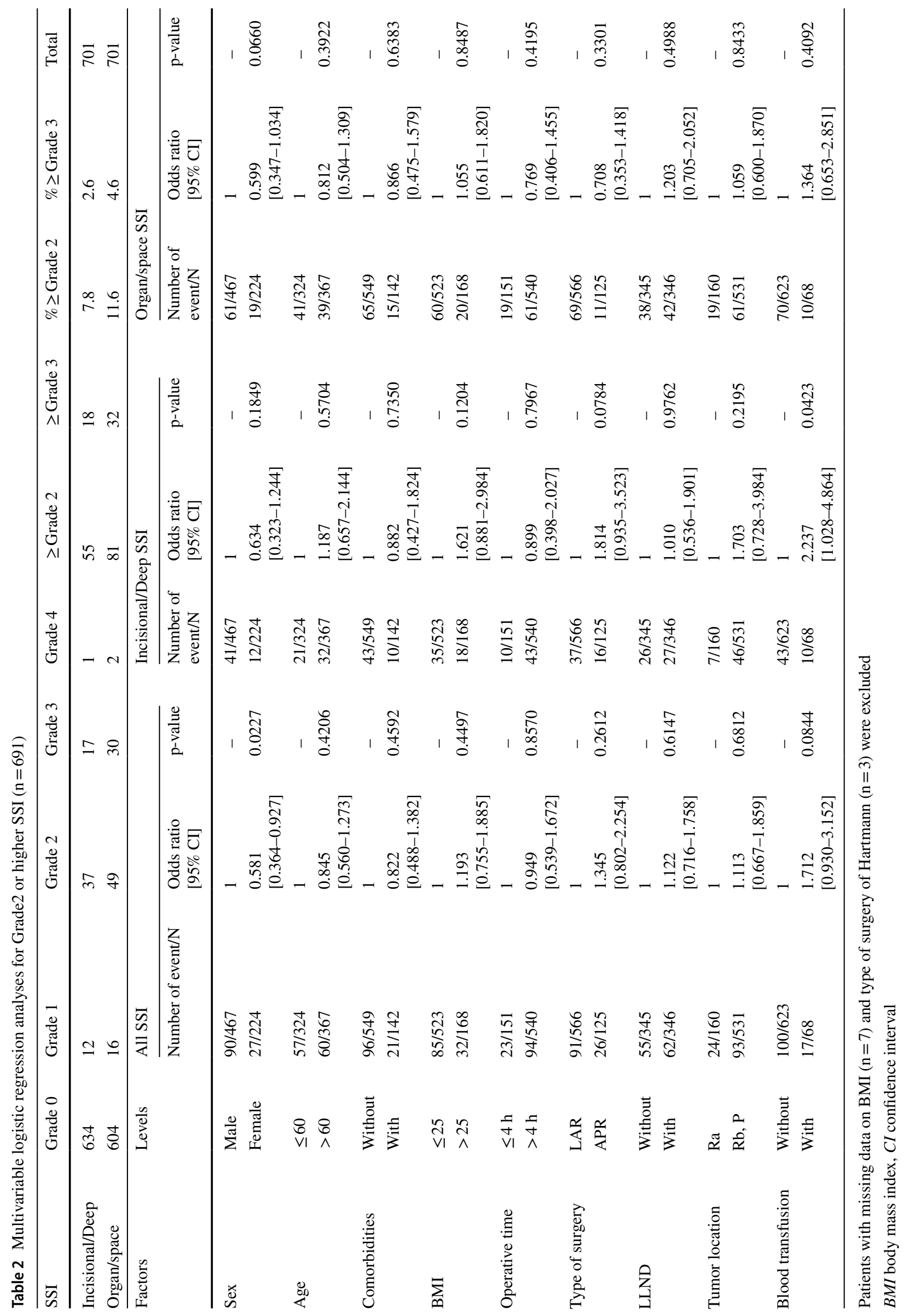



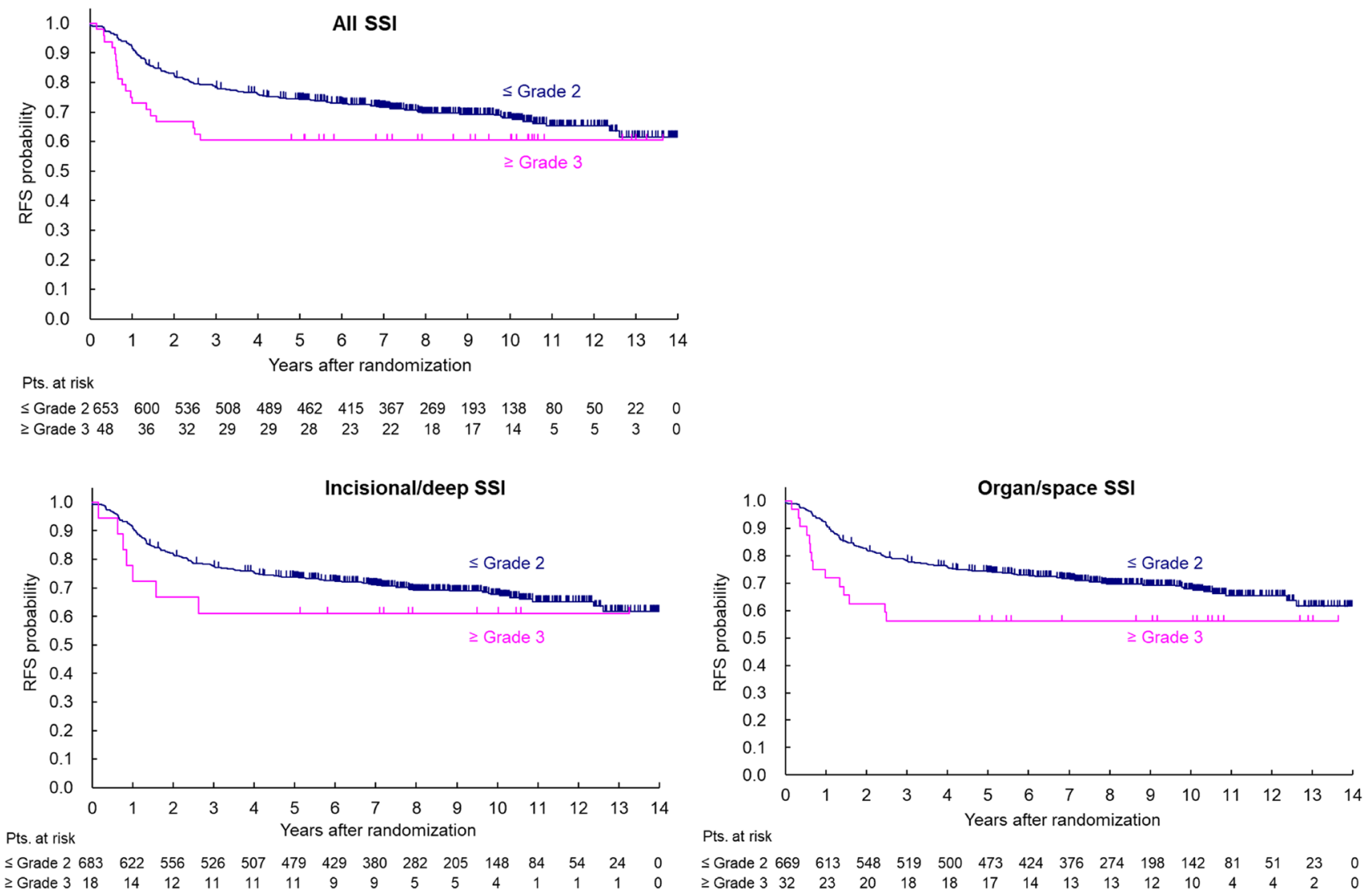

Fig. 1 Relapse-free survival curves (Grade 2 or lower vs. Grade 3 or higher SSI); all SSI (top figure), incisional/deep SSI (bottom left), and organ/space SSI (bottom right)

many reports have been published on the association of cancer recurrence with SSI. Associations with recurrence [7] and those with local recurrence of rectal cancer [16], wound infection, anastomotic leakage, and all infections have been reported [17]. Although the results have not yet been confirmed, most reports indicate an association between recurrence and prognosis. There have been various reports on these mechanisms, and delays of adjuvant chemotherapy, systemic inflammation and cytokines, and implantation of cancer cells, among others [18], [19] have been observed.

This study showed an association between all and organ/ space SSI and recurrence. Significant differences were recognized in patients with Grade 3 or higher SSI, and almost all patients experienced recurrence within 3 years. This suggested that in case of Grade 3 or higher SSI, patients have a systemic response that may affect cancer progression. This study suspected that Grade 3 or higher SSI induced poor prognosis in patients with systemic inflammatory responses, such as a high neutrophil-lymphocyte count ratio and high CRP, which has been shown in patients with advanced colorectal cancer [18]. However, it should be noted that Grade 3 or higher SSI were only reported in a limited number of patients (6.8\% of the total) and this should be considered in the interpretation of the results.

Conversely, local RFS curves were similar and no effects were observed on local recurrence in this study. The incidence of SSI has negative effects on both medical economics and prognoses, and thus, sufficient countermeasures are necessary.

In addition, the 7-year overall survival (OS) for patients with Grade 2 or lower and Grade 3 or higher all SSI were $82.1 \%$ and $79.1 \%$. For incisional/deep SSI, the 7-year OS for patients with Grade 2 or lower and Grade 3 or higher SSI were $82.4 \%$ and $77.8 \%$. For organ/space SSI, the 7-year OS in patients with Grade 2 or lower and Grade 3 or higher SSI were $82.0 \%$ and $78.2 \%$. Grade 3 or higher SSI did not affect the overall survival rate.

But this study has some limitations. One is that there were few events of SSI. Another one is that the primary endpoint of this clinical trial was not the association of SSI. And therefore we might not take enough dates for analysis.

In conclusion, the risk factors of the incidence of Grade 2 or higher all SSI was male sex and that of incisional/deep SSI was presence of blood transfusions. LLND was not associated with any SSI. The incidence of Grade 3 or higher all 


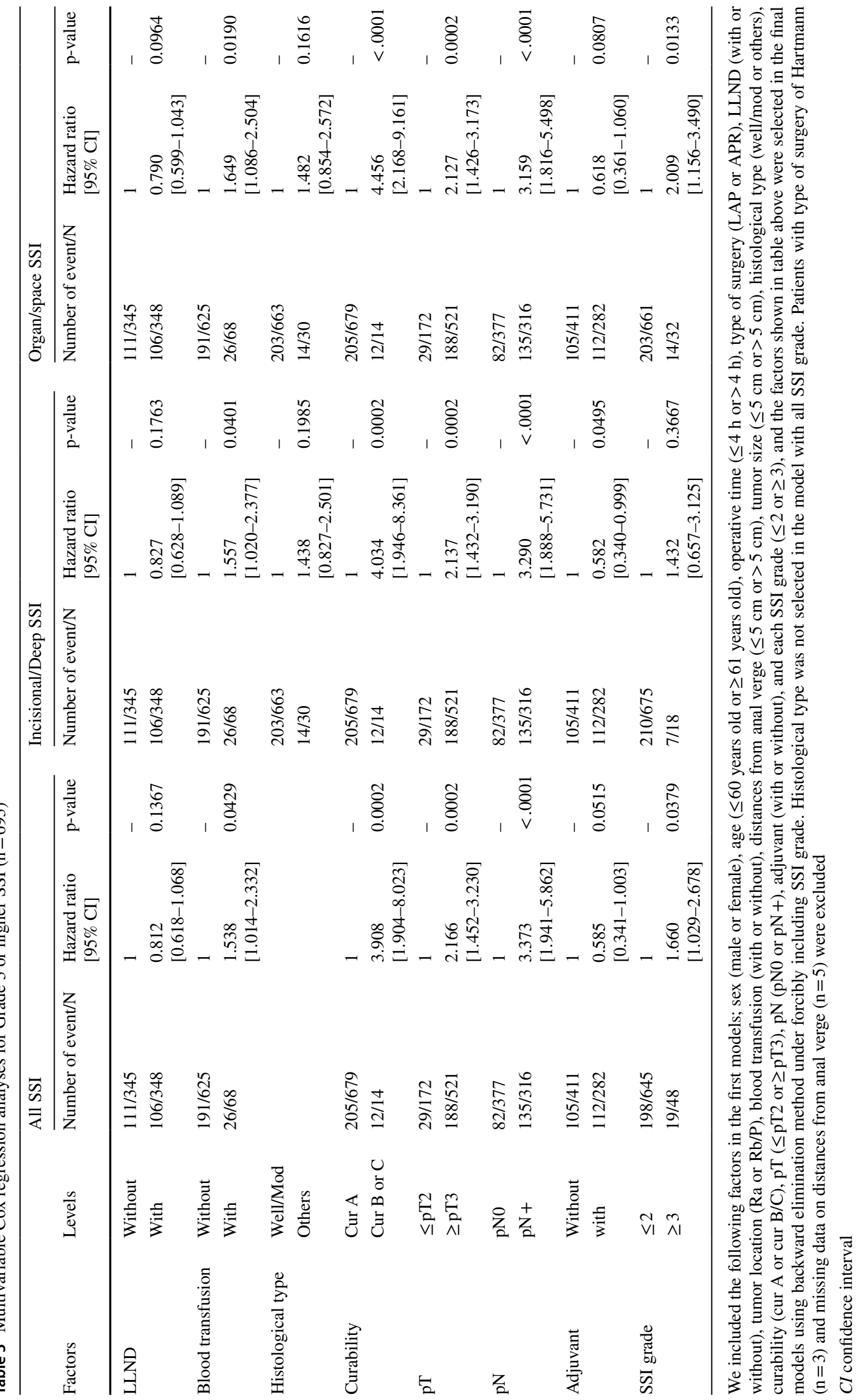


SSI and space/organ SSI was a significant prognostic factor in laparotomy for rectal cancer and requires preventive measures.

Acknowledgements This study was supported in part by the National Cancer Center Research and Development Fund and Grants-in-Aid for Cancer Research, Health Sciences Research Grants for Medical Frontier Strategy Research, Health and Labor Sciences Research Grants for Clinical Research for Evidence-based Medicine, and Health and Labor Sciences Research Grant for Clinical Cancer Research from the Ministry of Health, Labour and Welfare of Japan. The authors are grateful to the members of the JCOG Data Center and Operations Office for their support in managing the data and overseeing study management (Haruhiko Fukuda).

\section{Declarations}

Conflict of interest The authors declare that they have no conflict of interest.

Open Access This article is licensed under a Creative Commons Attribution 4.0 International License, which permits use, sharing, adaptation, distribution and reproduction in any medium or format, as long as you give appropriate credit to the original author(s) and the source, provide a link to the Creative Commons licence, and indicate if changes were made. The images or other third party material in this article are included in the article's Creative Commons licence, unless indicated otherwise in a credit line to the material. If material is not included in the article's Creative Commons licence and your intended use is not permitted by statutory regulation or exceeds the permitted use, you will need to obtain permission directly from the copyright holder. To view a copy of this licence, visit http://creativecommons.org/licenses/by/4.0/.

\section{References}

1. Allegranzi B, Bagheri NS, Combescure C et al (2011) Burden of endemic health-care associated infection in developing countries: systematic review and meta-analysis. Lancet 377:228-241

2. Ueno M, Oya M, Azekura K et al (2005) Incidence and prognostic significance of lateral lymph node metastasis in patients with advanced low rectal cancer. Br J Surg 92:756-763

3. Sugihara K, Kobayashi H, Kato T et al (2006) Indication and benefit of pelvic sidewall dissection for rectal cancer. Dis Colon Rectum 49:1663-1672

4. World health Organization (2016) Global guidelines for the prevention of surgical site infection. https://www.who.int/infectionprevention/en/

5. Surgical Site Infection (SSI) Guideline for Prevention of Surgical site Infection (2017) Centers https://www.cdc.gov/for Disease Control and Prevention, Guideline for the Prevention of Surgical Site Infection. https://www.cdc.gov/f
6. Japan Nosocomial Infections Surveillance (JANIS) (2013) http:// www.nih-janis.jp/report/ssi.html. Accessed on May 31, (2013)

7. Park EJ, Baik SH, Kang J et al (2016) The impact of postoperative complications on long-term oncologic outcomes after laparoscopic low anterior resection for rectal cancer. Medicine 95:e3271

8. Watanabe T, Muro K, Ajioka Y et al (2018) Japanese Society for Cancer of the Colon and Rectum (JSCCR) guidelines 2016 for the treatment of colorectal cancer. Int J Clin Oncol 23:1-34

9. Moriya Y, Sugihara K, Akasu T et al (1995) Nerve-sparing surgery with lateral node dissection for advanced lower rectal cancer. Eur J Cancer 31:1229-1232

10. Fujita S, Mizusawa J, Kanemitsu Y et al (2017) Mesorectal excision with or without lateral lymph node dissection for clinical stage II/III lower rectal cancer (JCOG0212): a multicenter, randomized controlled, noninferiority trial. Ann Surg 266:201-207

11. Fujita S, Akasu T, Mizusawa J et al (2012) Postoperative morbidity and mortality after mesorectal excision with and without lateral lymph node dissection for clinical Stage II or Stage III lower rectal cancer (JCOG0212): results from a multicentre, randomised controlled, non-inferiority trial. Lancet Oncol 13:616-621

12. National Cancer Institute-Common Toxicity Criteria (NCI-CTC Version 2.0, April 30, 1999) CTC Japanese translation Japan Clinical Oncology Group 1999.

13. Kato K, Igaki H, Ito Y et al (2019) Parallel-group controlled trial of esophagectomy versus chemoradiotherapy in patients with clinical Stage I esophageal carcinoma (JCOG0502)JCOG 0502. J Clin Oncol 37(4-suppl):7

14. Kobayashi M, Takesue Y, Kitagawa Y et al (2009) Current perioperative practice in patients with colorectal surgery: results of a questionnaire survey in Japan. J Jpn Soc Surg Infect 6:587-594

15. Sutton E, Miyagaki H, Bellini G et al (2017) Risk factors for superficial surgical site infection after elective rectal cancer resection: multivariate analysis of 8880 patients from the American College of Surgeons National Surgical Quality Improvement Program database. J Surg Res 207:205-214

16. Fujita S, Teramoto T, Watanabe M et al (1993) Anastomotic leakage after colorectal cancer surgery: a risk factors for recurrence and poor prognosis. Jpn J Clin Oncol 23:299-302

17. Mirnezami A, Mirnezami R, Chandrakumaran K et al (2011) Increased local recurrence and reduced survival from colorectal cancer following anastomotic leak. Ann Surg 253:890-899

18. Li Z, Zhao R, Cui Y et al (2018) The dynamic change of neutrophil to lymphocyte ratio can predict clinical outcome in stage I-III colon cancer. Sci Rep 8:9453

19. Krarup PM, Nordholm-Carstensen A, Jorgensen LN et al (2014) Anastomotic leak increases distant recurrence and long-term mortality after curative resection for colonic cancer: a nationwide cohort study. Ann Surg 259:930-938

Publisher's Note Springer Nature remains neutral with regard to jurisdictional claims in published maps and institutional affiliations. 


\section{Authors and Affiliations}

Kenji Katsumata ${ }^{1}$ - Masanobu Enomoto ${ }^{1} \cdot$ Tetsuo Ishizaki $^{1} \cdot$ Shin Fujita $^{2} \cdot$ Yukihide Kanemitsu $^{3}$ - Masaaki Ito ${ }^{4}$. Akio Shiomi ${ }^{5} \cdot$ Koji Komori $^{6} \cdot$ Masayuki Ohue $^{7} \cdot$ Mitsuyoshi Ota $^{8} \cdot$ Yoshihiro Akazai $^{9} \cdot$ Manabu Shiozawa $^{10}$. Takashi Yamaguchi $^{11}$. Hiroyuki Bando ${ }^{12}$. Mitugu Sekimoto ${ }^{13} \cdot$ Takaya Kobatake $^{14} \cdot$ Ryunosuke Machida $^{15}$. Takayuki Akasu $^{16}$. Yoshihiro Moriya ${ }^{17}$. the Colorectal Cancer Study Group of Japan Clinical Oncology Group

1 Department of Gastrointestinal and Pediatric Surgery, Tokyo Medical University, 6-7-1, Nishi-Shinjyuku Shinjyuku-ku, Tokyo 160-0023, Japan

2 Department of Surgery, Tochigi Cancer Center, Tochigi, Japan

3 Department of Colorectal Surgery, National Cancer Center Hospital, Tokyo, Japan

4 Department of Colorectal Surgery, National Cancer Center Hospital East, Chiba, Japan

5 Division of Colon and Rectal Surgery, Shizuoka Cancer Center Hospital, Shizuoka, Japan

6 Department of Surgery, Aichi Cancer Center Hospital, Nagoya, Japan

7 Department of Gastroenterological Surgery, Osaka International Cancer Institute, Osaka, Japan

8 Department of Surgery, Yokohama City University Medical Center, Kanagawa, Japan

9 Department of Surgery, Okayama Saiseikai General Hospital, Okayama, Japan
10 Department of Surgery, Kanagawa Cancer Center, Yokohama, Japan

11 Department of Surgery, Kyoto Medical Center, Kyoto, Japan

12 Department of Surgery, Ishikawa Prefectural Central Hospital, Ishikawa, Japan

13 Department of Surgery, National Hospital Organization Osaka National Hospital, Osaka, Japan

14 Department of Surgery, Shikoku Cancer Center, Ehime, Japan

15 JCOG Data Center and Operations Office, National Cancer Center Hospital, Tokyo, Japan

16 Department of Surgery, The Imperial Household Agency Hospital, Tokyo, Japan

17 Department of Surgery, Japanese Red Cross Medical Center, Tokyo, Japan 